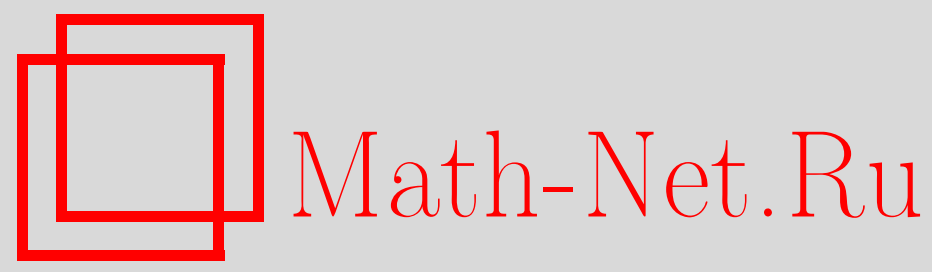

А. В. Шаповалов, Совместность и алгоритм распознавания несовместности реализаций случайных систем дискретных уравнений с двузначными неизвестными, Дискрет. матем., 2008, том 20, выпуск 3, 28-39

DOI: https://doi.org/10.4213/dm1010

Использование Общероссийского математического портала Math-Net.Ru подразумевает, что вы прочитали и согласны с пользовательским соглашением http://www . mathnet.ru/rus/agreement

Параметры загрузки:

IP: 54.84 .234 .179

26 апреля 2023 г., $17: 50: 55$

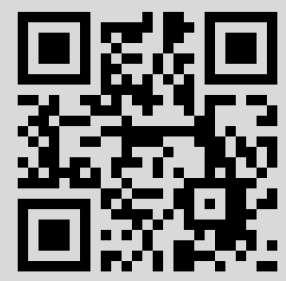




\title{
Совместность и алгоритм распознавания несовместности реализаций случайных систем дискретных уравнений с двузначными неизвестными
}

\author{
() 2008 г. А. В. Шаповалов
}

\begin{abstract}
Рассматривается случайная система дискретных уравнений относительно $n$ двузначных неизвестных, состоящая из $M=M(n)$ уравнений. Функции в уравнениях выбираются случайно из конечного множества функций и могут зависеть не более чем от $m$ переменных. Обоснован критерий наличия у случайной системы уравнений пороговой функции совместности, определяемой как функция $Q(n)$, для которой вероятность совместности случайной системы уравнений стремится к единице и к нулю при $n \rightarrow \infty, M(n) / Q(n) \rightarrow 0$ и $M(n) / Q(n) \rightarrow \infty$. Показано, что пороговые функции совместности могут иметь только вид $n$ и $n^{1-1 / r}, 2 \leqslant r \leqslant m+1$; построены критерии наличия у случайной системы уравнений таких пороговых функций. Для случайных систем уравнений с пороговыми функциями вида $n^{1-1 / r}, 2 \leqslant r \leqslant m+1$, оценена вероятность совместности при $M \sim c n^{1-1 / r}, n \rightarrow \infty$ (она убывает от единицы до нуля, принимая все промежуточные значения, с ростом $c$ от нуля до $\infty$ ) и построен алгоритм распознавания несовместности реализаций случайных систем уравнений. Этот алгоритм имеет такую же предельную вероятность определения несовместности систем уравнений, как и алгоритм полного перебора решений, но низкую трудоемкость порядка $n^{1-1 / r}$.
\end{abstract}

\section{1. Основные определения и результаты}

Пусть $\Phi_{i}=\left\{f_{i, 1}, f_{i, 2}, \ldots, f_{i, m_{i}}\right\}$ - упорядоченное множество функций, заданных на множестве $i$-мерных векторов, координаты которых выбираются из множества $B=\{0,1\}$, $m_{i}=\left|\Phi_{i}\right|, i=0,1, \ldots, m$. Каждая функция из $\Phi_{i}$ существенно зависит ровно от $i$ переменных и принимает значения в конечном множестве $A=\left\{a_{0}, \ldots, a_{q-1}\right\}, q>1$. Декартово произведение трех множеств $\{0,1, \ldots, m\},\left\{1, \ldots, \max _{i=0,1, \ldots, m} m_{i}\right\}$ и $A$ обозначим через $E$. Пусть $\bar{c}=\left\{c_{i, j, a},(i, j, a) \in E\right\}-$ набор неотрицательных констант, сумма которых равна единице, и $c_{i, j, a}=0$, если $j>m_{i}$ или $a$ не принадлежит множеству значений функции $f_{i, j}$. Через $\bar{b}$ будем обозначать второй элемент из $B$.

Случайная система уравнений $S=S_{n, M, \bar{c}}$ относительно $n$ неизвестных $x_{1}, \ldots, x_{n}$ состоит из $M$ уравнений, которые выбираются последовательно, случайно и независимо, вероятность появления уравнения вида

$$
f_{i, j}\left(x_{s_{1}}, x_{s_{2}}, \ldots, x_{s_{i}}\right)=a
$$


равна $c_{i, j, a}$ для каждого набора $(i, j, a) \in E$, а выбор упорядоченного набора индексов $s_{1}, \ldots, s_{i}$ из множества $\{1, \ldots, n\}$ осуществляется независимо, случайно и равновероятно из всех $n(n-1) \ldots(n-i+1)$ возможных упорядоченных наборов по $i$ штук (выборка без возвращения). Индексы $i$ и $j$ в (1) означают, что функция в левой части уравнения является $j$-м элементом множества $\Phi_{i}$.

В работе исследуется асимптотическое поведение вероятности совместности случайной системы уравнений $S$ при $n \rightarrow \infty$, когда число ее уравнений $M=M(n)$ зависит от $n$. Все асимптотические формулы приводятся при условии, что $n \rightarrow \infty$, используются обозначения $(k)_{i}=k(k-1) \ldots(k-i+1),\lfloor x\rfloor$ - целая часть числа $x$.

Основные используемые определения приводятся ниже, остальные можно найти в $[4,12-15]$. Под свойством $Q=Q_{n}$ случайного элемента (случайного гиперграфа или случайной системы уравнений) понимается некоторое подмножество его множества реализаций. Под вероятностью свойства $Q$ случайного элемента понимается сумма вероятностей реализаций этого случайного элемента, входящих в $Q$. Вероятность совместности $P_{c}(S)$ случайной системы уравнений $S$ равна сумме вероятностей совместных реализаций этой случайной системы уравнений. При заданном соотношении параметров $n$ и $M(n)$ почти все реализации случайного элемента обладают свойством $Q$, если вероятность этого свойства стремится к единице при $n \rightarrow \infty$. Если существует функция $Q(n)$, возрастающая при $n \rightarrow \infty$ и такая, что $P_{c}(S)$ стремится к единице (нулю) при $M(n) / Q(n) \rightarrow 0$ $(M(n) / Q(n) \rightarrow \infty)$, то она называется пороговой функцией совместности случайной системы уравнений $S$. Минимальной несовместной подсистемой системы уравнений будем называть несовместную подсистему, которая становится совместной при исключении из нее любого уравнения, допустимой подсистемой - подсистему, состоящую из уравнений вида (1), для которых $c_{i, j, a}>0$. Концевым ребром гиперграфа называется ребро, все вершины которого, кроме, может быть, одной, инцидентны только этому ребру. Связный гиперграф без циклов называется гипердеревом.

Для каждого набора $(i, j, a) \in E$ обозначим через $t_{i, j, a}^{(0)}$ и $t_{i, j, a}^{(1)}$ числа однозначно определяющихся из уравнения (1) переменных, равных 0 и 1. Под подфункцией $g_{s_{1}, \ldots, s_{t}}^{b_{1}, \ldots, b_{t}}\left(y_{1}, \ldots, y_{i}\right)$ функции $g\left(y_{1}, \ldots, y_{i}\right)$ обычно понимается функция $i-t$ переменных, получаемая из функции $g\left(y_{1}, \ldots, y_{i}\right)$ в результате фиксации переменных с номерами $s_{1}, \ldots, s_{t}$ значениями $b_{1}, \ldots, b_{t}$. Скажем, что множество $W=\left\{s_{1}, \ldots, s_{t}\right\} \subseteq\{1, \ldots, i\}$ является несовместной фиксацией ( $t$-фиксацией) значением $b$ переменных уравнения (1), где $1 \leqslant t \leqslant i, b \in B$, если при $g=f_{i, j}$ уравнение $g_{s_{1}, \ldots, s_{t}}^{b, \ldots, b}\left(y_{1}, \ldots, y_{i}\right)=a$ несовместно. Для каждого набора $(i, j, a) \in E$ обозначим через $k_{i, j, a}^{(b, t)}$ число несовместных $t$-фиксаций значением $b$ переменных уравнения (1).

Среднее значение $c^{(b)}$ чисел, однозначно определяющихся из уравнения случайной системы уравнений $S$ переменных, равных $b$, и среднее значение $c^{(b, t)}$ числа несовместных $t$-фиксаций значением $b$ уравнения этой системы удовлетворяют формулам

$$
c^{(b)}=\sum_{(i, j, a) \in E} c_{i, j, a} t_{i, j, a}^{(b)}, \quad c^{(b, t)}=\sum_{(i, j, a) \in E, t \leqslant i \leqslant m} c_{i, j, a} k_{i, j, a}^{(b, t)}, \quad b \in\{0,1\} .
$$

Рангом несовместных фиксаций значением $b$ переменных уравнения (1) назовем минимальное значение $t$, при котором существует хотя бы одна несовместная фиксация значением $b$ переменных такого уравнения. Если $f_{i, j}(b, \ldots, b)=a$, то этот ранг равен нулю. Обозначим через $R^{(b)}$ ранг несовместных фиксаций значением $b$ переменных уравнения случайной системы $S$, равный минимальному значению $r$, при котором $c^{(b, r)}>0$. Если $c^{(b, r)}=0$ для каждого $r$ из множества $\{1, \ldots, m\}$, то $R^{(b)}=0$. 
Рассмотрим алгоритм Al распознавания несовместности реализаций случайной системы уравнений $S$ с пороговой функцией совместности $n^{1-1 / r}, r=2, \ldots, m+1$.

Алгоритм имеет два шага.

1. Из уравнений с однозначно определяющимися переменными выявляются все равные $b$ неизвестные системы уравнений.

2. Перебираются уравнения с несовместными $(r-1)$-фиксациями значением $b$. Если найдется такое уравнение с фиксацией $\left\{s_{1}, \ldots, s_{t}\right\}$, что в нем на местах $s_{1}, \ldots, s_{t}$ стоят неизвестные, значения которых на первом шаге были определены (и равны $b$ ), то система уравнений несовместна; в противном случае не делается никакого вывода о ее совместности.

Через $\pi=\pi(\mathrm{Al})$ обозначим надежность алгоритма $\mathrm{Al}$, определяемую как сумму вероятностей реализаций случайной системы уравнений $S$, несовместность которых этот алгоритм распознает.

Теорема 1. Случайная система уравнений $S$ имеет пороговую функиию совместности $n^{1-1 / r}, r=2, \ldots, m+1$, тогда и только тогда, когда для некоторого $b \in B$ выполняются соотношения $c^{(b)}>0, R^{(b)}=r-1$, в этом случае при

$$
M \sim c n^{1-1 / r}
$$

где с - положительная постоянная, справедливы оиенки

$$
P_{c}(S) \sim e^{-\lambda}, \quad \pi \sim 1-e^{-\lambda}, \quad \lambda=c^{r} c^{(b, r-1)}\left(c^{(b)}\right)^{r-1} .
$$

Теорема 2. Случайная система уравнений $S$ имеет пороговую функиию совместности $n$ тогда и только тогда, когда $c^{(0)}+c^{(1)}=0, R^{(0)} R^{(1)}>0$.

Теорема 3. Случайная система уравнений $S$ имеет пороговую функиию совместности тогда и только тогда, когда $R^{(0)} R^{(1)}>0$. Пороговая функиия совместности случайной системы уравнений $S$ равна $n$ или $n^{1-1 / r}, r=2, \ldots, m+1$.

В условиях теоремы 1 надежность алгоритма Al эквивалентна определяемой аналогично надежности алгоритма полного перебора решений, но алгоритм Al при условии (2) имеет трудоемкость порядка $n^{1-1 / r}$, а трудоемкость алгоритма перебора - экспоненциального порядка. При $R^{(0)}=0$ и/или $R^{(1)}=0$ случайная система уравнений $S$ имеет решение $\mathbf{0}=(0, \ldots, 0)$ и/или $\mathbf{1}=(1, \ldots, 1)$ и не имеет других решений. Условие $R^{(0)} R^{(1)}>0$ эквивалентно существованию двух наборов $\left(i_{0}, j_{0}, a_{0}\right),\left(i_{1}, j_{1}, a_{1}\right)$, удовлетворяющих условиям

$$
f_{i_{0}, j_{0}}(0, \ldots, 0) \neq a_{0}, \quad f_{i_{1}, j_{1}}(1, \ldots, 1) \neq a_{1}, \quad c_{i_{0}, j_{0}, a_{0}} c_{i_{1}, j_{1}, a_{1}}>0 .
$$

Случайная система уравнений $S$ является частным случаем изучавшейся в [13] случайной системы уравнений, для которой $|B| \geqslant 2$. Теорема 1 , в отличие от близких по форме теоремы 4.1 работы [13] и формулы (2.5) статьи [2], конструктивна, то есть дает конкретный вид оценок вероятностей совместности случайных систем уравнений. В теореме 3 показано, что при $|B|=2$ параметр $r$ у пороговой функции совместности $n^{1-1 / r}$ не превосходит $m+1$ и доказан критерий наличия пороговой функции совместности. Понятие пороговой функции совместности случайной системы уравнений введено в [12], где показана ее связь с пороговой функцией свойства случайных графов и гиперграфов, 
заключающегося в наличии подграфов заданного вида (см. также [13-15]). Алгоритмы распознавания несовместности реализаций случайных систем уравнений с пороговыми функциями $\sqrt{n}, n^{2 / 3}$ и одной функцией в левых частях уравнений исследовались Г. В. Балакиным и В. А. Копытцевым. Результаты и обзоры по данной тематике можно найти в $[1-3,5-13]$.

\section{2. Геометрические свойства случайной системы уравнений}

Для изучения геометрических свойств реализаций случайной системы уравнений ей сопоставляется случайный гиперграф (см. [5, 7, 12, 13]). Каждой реализации случайной системы уравнений $S$ сопоставляется гиперграф. При этом переменной $x_{j}$ соответствует вершина $v_{j}, j=1, \ldots, n$, а каждое уравнение системы однозначно определяет соответствующее ребро сопоставляемого системе гиперграфа: ребро состоит из упорядоченных вершин, соответствующих существенным переменным функции в уравнении. Порядок на множестве вершин определяется порядком на множестве неизвестных соответствующего уравнения. Каждое ребро имеет метку, выбираемую из множества $E$. Метка ребра гиперграфа равна $(i, j, a)$ тогда и только тогда, когда в левой части соответствующего ему уравнения стоит функция $f_{i, j}$, а в правой части этого уравнения стоит элемент $a$. Случайной системе уравнений $S$ сопоставляется случайный гиперграф $M G(S)$, реализациями которого являются все гиперграфы с множеством вершин $\left\{v_{1}, \ldots, v_{n}\right\}$ и $M$ упорядоченными ребрами. Вершины в ребрах упорядочены, ребра имеют метки из множества $E$. Вероятности реализаций случайного гиперграфа $M G(S)$ равны вероятностям соответствующих им реализаций случайной системы уравнений $S$. Случайная система уравнений $S$ и соответствующий ей случайный гиперграф однозначно определяют друг друга.

Справедливы следующие эквивалентные определения случайной системы уравнений $S$ и случайного гиперграфа $M G(S)$. Последовательно, независимо друг от друга, с вероятностью $c_{i}=\sum_{(j, a)} c_{i, j, a}$, для указанных случайных элементов выбирается число $i$ существенных переменных в уравнении (вершин в ребре), $i=0,1, \ldots, m, c_{0}+\ldots+c_{m}=1$. Для каждого уравнения, зависящего от $i$ переменных (ребра из $i$ вершин), случайно, равновероятно и независимо от других уравнений (ребер) выбираются $i$ неупорядоченных неизвестных (вершин), $i=0,1, \ldots, m$, затем они случайно и равновероятно упорядочиваются одним из $i$ ! возможных вариантов. После этого для каждого уравнения, зависящего от $i$ переменных (ребра из $i$ вершин), независимо от других уравнений (ребер) с вероятностью $c_{i, j, a} / c_{i}$ для всех пар $(j, a)$ выбирается функция $f_{i, j}$ из $\Phi_{i}$ и правая часть $a$ из множества $A$ (вторая и третья компоненты метки ребра).

Такие определения случайных элементов позволяют сделать вывод, что для первоначального изучения их геометрической структуры необязательно знать весь набор $\bar{c}$ и порядок на множествах вершин в ребрах. Знание величин $c_{0}, \ldots, c_{m}$ уже позволяет исследовать эту структуру (наличие гипердеревьев и циклов, их вид и др.) без детализации, учитывающей конкретный вид функций и правые части уравнений. Если в последнем из приведенных определений случайного гиперграфа $M G(S)$ остановиться после выбора неупорядоченных вершин для ребер его реализаций, то получится случайный гиперграф $M G_{n, \bar{c}}$, свойства которого изучались в $[12,13]$. Следовательно, эти результаты описывают основные черты докритического этапа эволюции и случайного гиперграфа $M G(S)$, в частности, почти все реализации случайного гиперграфа при $M=o(n)$ не имеют циклов, а при $M=o\left(n^{1-1 / r}\right)$ - гипердеревьев из $r$ и более ребер. 
Докажем две леммы.

Лемма 1. Пусть $\Phi=\left\{\Phi_{0} \cup \ldots \cup \Phi_{m}\right\}$ и $U$-некоторое множество совместных уравнений с функциями из множества Ф. Тогда

(a) если $U$ содержит уравнение с однозначно определяющейся переменной, равной $b$, $b \in B, u$ минимальное значение ранга несовместных фиксаџий значением $b$ переменных уравнений из множества $U$ равно $t-1,2 \leqslant t \leqslant m+1$, то из элементов $U$ можно построить несовместные подсистемь уравнений, соответствующие гипердеревьям, причем минимальное значение числа уравнений в таких подсистемах равно $t$, и любая минимальная несовместная подсистема из $t$ уравнений имеет следующий вид:

(1) функция в левой части $t$-го уравнения имеет несовместную $(t-1)$-фиксацию $W=\left\{s_{1}, \ldots, s_{t-1}\right\}$ значением $b$ переменных этого уравнения,

(2) первые $t$ - 1 уравнений попарно не имеют общих переменных, но каждое из них имеет ровно одну общую переменную с $t$-м уравнением, которая в $k$-м уравнении однозначно определяется и равна $b$, а в $t$-м уравнении имеет номер $s_{k}, s_{k} \in W, k=1, \ldots, t-1$;

(б) если в $U$ нет уравнений с несовместной фиксаџией элементом $b \in B$ их переменных, то любая система уравнений, состоящая из элементов $U$, совместна и имеет решение $\mathbf{b}=(b, \ldots, b)$.

Доказательство. Отсутствие несовместных фиксаций значением $b$ переменных уравнения означает, что при фиксации всех его переменных значением $b$ оно остается совместным и имеет решение b. Таким свойством обладают все уравнения в $U$. Из этого следует утверждение (б) леммы.

Перейдем к утверждению (а) леммы. Пусть минимальное значение ранга $t$ несовместных фиксаций значением $b$ переменных уравнений из множества $U$ равно единице. Это равносильно существованию уравнения с однозначно определяющейся переменной, равной $\bar{b}$. Тогда из элементов $U$ можно построить описанную в условиях 1,2 подсистему из двух уравнений, соответствующую гипердереву. Она состоит из двух уравнений с одной общей переменной, которая однозначно определяется из каждого уравнения, причем ее значения равны $b$ и $\bar{b}$ соответственно. Минимальность такой подсистемы обеспечивается совместностью входящих в нее уравнений, выбираемых из $U$.

Пусть теперь минимальное значение $t$ ранга несовместных фиксаций значением $b$ переменных уравнений из множества $U$ больше единицы. Это означает отсутствие в $U$ уравнений с однозначно определяющимися переменными, равными $\bar{b}$. Подсистема уравнений, определяемая условиями 1,2 , несовместна, соответствует гипердереву и состоит из уравнений, принадлежащих множеству $U$. Покажем, что каждая такая подсистема из $t$ уравнений минимальна, и что любая минимальная несовместная подсистема из $t$ элементов $U$ имеет такой же вид.

Пусть несовместная подсистема $S(H)$ минимальна, соответствует гипердереву $H$ и состоит из минимально возможного числа $k$ уравнений. Очевидно, что $2 \leqslant k \leqslant t$. Любое гипердерево из двух или более ребер имеет концевое ребро. Пусть уравнение $u_{1}$ подсистемы $S(H)$ в гипердереве $H$ соответствует концевому ребру. Уравнение $u_{1}$ имеет ровно одну общую переменную с остальными уравнениями подсистемы, которая из уравнения $u_{1}$ однозначно определяется и равна $b$. Последнее следует из минимальности подсистемы уравнений, так как в противном случае (переменная не определяется однозначно) 
исключение уравнения $u$ из подсистемы дает несовместную подсистему с меньшим, чем $t$, числом уравнений. Обозначим через $u_{0}$ уравнение подсистемы $S(H)$, которое имеет общую переменную с уравнением $u_{1}$ и которому в гиперграфе $H$ соответствует ребро, не являющееся концевым. Хотя бы одно такое уравнение существует, так как в противном случае подсистема $S(H)$ состояла бы из $k$ уравнений, имеющих одну общую переменную, и была бы совместной ввиду отсутствия в $U$ уравнений с однозначно определяющимися переменными, равными $\bar{b}$.

Через $S\left(H^{(0)}\right)$ обозначим подсистему, составленную из уравнения $u_{0}$ и всех уравнений подсистемы $S(H)$, которые имеют общие неизвестные с этим уравнением и в гиперграфе $H$ соответствуют концевым ребрам. Подсистема $S\left(H^{(0)}\right)$, вообще говоря, может иметь общие неизвестные с остальными уравнениями подсистемы $S(H)$. Не теряя общности, обозначим одно такое неизвестное $x_{1}$. Через $S\left(H^{(1)}\right)$ обозначим соответствующую гипердереву подсистему, составленную из всех уравнений подсистемы $S(H)$, которые не входят в $S\left(H^{(0)}\right)$ и одно из которых содержит неизвестное $x_{1}$. Подсистема $S\left(H^{(1)}\right)$ либо пуста, либо содержит не менее двух уравнений, так как в противном случае единственное ее уравнение соответствует висячему ребру в гиперграфе $H$ и должно принадлежать подсистеме $S\left(H^{(0)}\right)$. В первом случае выполняется утверждение леммы. Методом от противного покажем, что второй случай невозможен. Из способа образования подсистемы $S\left(H^{(1)}\right)$ следует, что она имеет с остальными уравнениями подсистемы $S(H)$ только одно общее неизвестное $x_{1}$. В подсистеме $S\left(H^{(1)}\right)$ неизвестное $x_{1}$ может: быть однозначно определено и равно $b$; быть однозначно определено и равно $\bar{b}$; не иметь ограничений. Во всех этих случаях на основе подсистемы $S(H)$ можно получить несовместную подсистему, соответствующую гипердереву и имеющую меньше $k$ уравнений: в первом случае заменив в $S(H)$ подсистему $S\left(H^{(1)}\right)$ на одно уравнение с однозначно определяющейся переменной $x_{1}$; во втором случае - заменив в $S(H)$ подсистему $S\left(H^{(0)}\right)$ на одно уравнение с однозначно определяющейся переменной $x_{1}$; в третьем случае - удалив подсистему $S\left(H^{(1)}\right)$ из $S(H)$. Это противоречит минимальности несовместной подсистемы $S(H)$, соответствующей гипердереву. Следовательно, подсистема $S\left(H^{(1)}\right)$ пуста, а подсистема $S(H)$ состоит из $k, 2 \leqslant k \leqslant t$, уравнений, причем каждое из первых $k-1$ уравнений имеет ровно одну общую переменную с $k$-м уравнением.

Осталось уточнить характеристики общих переменных уравнений подсистемы $S(H)$ и ее геометрический вид. Выше показано, что $s$-е уравнение подсистемы $S(H)$ в гипердереве $H$ соответствует концевому ребру, и его общая с $k$-м уравнением переменная однозначно определяется из $s$-го уравнения и равна $b, s=1, \ldots, k-1$. Подсистема $S(H)$ соответствует гипердереву, поэтому любые два из этих первых $k-1$ уравнений могут иметь общую переменную только из числа принадлежащих $k$-му уравнению. Однако это противоречит минимальности подсистемы $S(H)$, так как если среди этих уравнений найдется пара с общей переменной, то одно из них можно удалить из подсистемы $S(H)$ с сохранением ее несовместности. Следовательно, первые $k-1$ уравнений попарно не имеют общих переменных. Для несовместности подсистемы $S(H)$ необходимо и достаточно, чтобы множество номеров переменных $k$-го уравнения, которые являются общими с переменными первого, второго, ..., $(k-1)$-го уравнений подсистемы, образовывало несовместную фиксацию этого уравнения. Минимальное значение ранга несовместных фиксаций значением $b$ переменных уравнений из множества $U$ равно $t-1$, поэтому $k=t$ и подсистема $S(H)$ удовлетворяет условиям 1,2 леммы.

Лемма 2. Пусть $H-$ совместная система уравнений с функциями из множества $\Phi \subseteq\left\{\Phi_{0} \cup \ldots \cup \Phi_{m}\right\}$; каждое из уравнений $U_{1}, \ldots, U_{k}$ совместно, имеет функиию из $\Phi$ и не имеет однозначно определяющихся переменных; $U_{i}$ имеет ровно одну общую пере- 
менную с $H \cup U_{1} \cup \ldots \cup U_{i-1}, i=1, \ldots, k$. Тогда система уравнений $H \cup U_{1} \cup \ldots \cup U_{k}$ совместна.

Доказательство. Выберем произвольное решение системы $H$ относительно входящих в нее переменных. Ввиду совместности $H$ это всегда возможно. Первое добавляемое уравнение $U_{1}$ имеет общую переменную $x_{j}$ с подсистемой $H$, которая в выбранном решении системы $H$ относительно входящих в нее переменных принимает некоторое значение $b$. Так как уравнение $U_{1}$ не имеет однозначно определяющихся переменных, оно имеет не менее одного решения, в котором переменная $x_{j}$ равна $b$. Следовательно, система уравнений $H_{1}=H \cup U_{1}$ совместна. Аналогично убеждаемся, что при поочередном добавлении остальных $k-1$ уравнений, имеющих с расширяемой системой ровно по одной общей переменной, получаем совместную систему.

\section{3. Доказательство основных результатов}

Доказательство теоремы 1. Из условий $c^{(b)}>0, R(b)=r-1$ доказываемой теоремы следует, что множество допустимых реализаций случайной системы уравнений $S$ удовлетворяет требованиям к множеству уравнений $U$ в условиях п. (а) леммы 1 , причем $t=r$. Достаточность условий наличия пороговой функции совместности следует из утверждения (а) леммы 1 и теоремы 3.1 статьи [13], в соответствии с которой случайная система уравнений $S$ имеет равную $n^{1-1 / r}, r \geqslant 2$, пороговую функцию совместности тогда и только тогда, когда в ее реализациях допустимы несовместные подсистемы, соответствующие гипердеревьям, причем минимальное число уравнений в таких подсистемах равно $r$.

Перейдем к обоснованию необходимости условий наличия пороговой функции совместности рассматриваемого вида у случайной системы уравнений $S$. Пусть $S$ имеет пороговую функцию совместности $n^{1-1 / r}$, где $r \in\{2, \ldots, m+1\}$. Тогда должно быть выполнено условие $c^{(0)}+c^{(1)}>0$. Действительно, почти все реализации случайного гиперграфа $M G(S)$ при условии $M=o(n)$ состоят из гипердеревьев. Из леммы 2 следует, что при условии $c^{(0)}+c^{(1)}=0$ подсистемы, соответствующие гипердеревьям, совместны. Поэтому при $M=o(n)$ и $c^{(0)}+c^{(1)}=0$ почти все реализации случайной системы уравнений $S$ совместны, что противоречит наличию у нее пороговой функции совместности, равной $n^{1-1 / r}, r \geqslant 2$. В случае $c^{(0)} c^{(1)}>0$, в соответствии с теоремой 4.1 статьи [13], случайная система уравнений $S$ имеет пороговую функцию совместности $\sqrt{n}$, а условие $c^{(0)} c^{(1)}>0$ эквивалентно условиям $r=2, c^{(b)}>0, R^{(b)}=1$ утверждения доказываемой теоремы. В оставшемся случае $c^{(0)}+c^{(1)}>0, c^{(0)} c^{(1)}>0$ для некоторого $b \in B$ справедливы соотношения $c^{(b)}>0, c^{(\bar{b})}=0$. Тогда ранг $R^{(b)}$ несовместных фиксаций значением $b$ переменных уравнения реализации случайной системы $S$ принадлежит множеству $\{1, \ldots, m\}$, так как в противном случае $R^{(b)}=0$, откуда, в соответствии с п. (б) леммы 1 , случайная система уравнений $S$ является заведомо совместной и не может иметь пороговую функцию совместности. Следовательно, выполнены условия п. (а) леммы 1 , и минимальные несовместные подсистемы реализаций случайной системы уравнений $S$ с минимальным числом уравнений, соответствующие гипердеревьям, состоят из $t$ уравнений вида, определяемого условиями 1, 2 п. (а) леммы 1. Отсюда и из теоремы 3.1 статьи [13] следует равенство $r=t$ и необходимость приведенных в утверждении теоремы 1 условий наличия пороговой функции совместности $n^{1-1 / r}, 3 \leqslant r \leqslant m+1$.

Осталось получить оценку вероятности совместности случайной системы уравнений $S$ при условии (2). В теореме 3.1 статьи [13] для случайной системы уравнений $S$, 
имеющей пороговую функцию совместности $n^{1-1 / r}$, при условии (2) получена оценка $P_{c}(S) \sim e^{-\lambda}$, где $\lambda=\lambda(c, r)$ - предельное значение среднего числа гипердеревьев, соответствующих несовместным подсистемам из $r$ уравнений в реализациях случайного гиперграфа $M G(S)$. Вид таких несовместных подсистем приведен в утверждении (a) леммы 1. Оценим величину $\lambda$. В левой части $r$-го уравнения таких подсистем стоит функция $f_{i_{k}, j_{k}}$, в правой - элемент $a_{k}, k=1, \ldots, r$, причем $c_{i_{k}, j_{k}, a_{k}} t_{i_{k}, j_{k}, a_{k}}^{(b)}>0$ для первых $r-1$ уравнений и $c_{i_{r}, j_{r}, a_{r}} k_{i_{r}, j_{r}, a_{r}}^{(b, r)}>0$ для последнего уравнения. Первые $r-1$ уравнений попарно не имеют общих переменных, но каждое их них имеет ровно одну общую переменную с $r$-ым уравнением. В каждом из этих $r-1$ уравнений общие с $r$-м уравнением переменные однозначно определяются и равны $b$, а номера совпадающих с ними переменных $r$-го уравнения образуют несовместную $t$-фиксацию значением $b$ уравнения (1) при $f_{i, j}=f_{i_{k}, j_{k}}$ и $a=a_{k}$. Из условий $c^{(b, r-1)}>0$ (так как $\left.R(b)=r-1\right)$ и $c^{(b)}>0$ следует, что множество подсистем такого вида не пусто. Несовместность таких подсистем показана в п. (а) леммы 1. Из условия $c_{i_{r}, j_{r}, a_{r}} \prod_{k=1}^{r-1} c_{i_{k}, j_{k}, a_{k}}>0$ следует, что они допустимы в реализациях рассматриваемой случайной системы уравнений.

Число таких гипердеревьев с учетом фиксированного порядка первых ребер равно $k_{i_{r}, j_{r}, a_{r}}^{(b, r)} \prod_{k=1}^{r-1} t_{i_{k}, j_{k}, a_{k}}^{(b)}$, а порядок группы автоморфизмов каждого такого гипердерева равен 1, так как вершины во всех ребрах упорядочены. В соответствии с теоремой 2.1 работы [13] предел среднего значения числа таких гипердеревьев в реализациях $M G(S)$ определяется формулой, в которой ведется суммирование по всем упорядоченным наборам $\left(i_{k}, j_{k}, a_{k}\right), k=1, \ldots, r$ :

$$
\begin{aligned}
& \left.c_{r} \sum_{\left(i_{r}, j_{r}, a_{r}\right)} c_{i_{r}, j_{r}, a_{r}} k_{i_{r}, j_{r}, a_{r}}^{(b, r-1)} \sum_{\substack{\left(i_{1}, j_{1}, a_{1}\right), \ldots,\left(i_{r-1}, j_{r-1}, a_{r-1}\right) \\
\left(c_{i_{1}, j_{1}, a_{1}}\right.}}^{(b)} t_{i_{1}, j_{1}, a_{1}}^{(b)} c_{i_{r-1}, j_{r-1}, a_{r-1} t} t_{i_{r-1}, j_{r-1}, a_{r-1}}^{(b)}\right) \\
& =c_{r} \sum_{\left(i_{r}, j_{r}, a_{r}\right)} c_{i_{r}, j_{r}, a_{r}} k_{i_{r}, j_{r}, a_{r}}^{(b, r-1)}\left(\sum_{(i, j, a) \in E} c_{i, j, a} t_{i, j, a}^{(b)}\right)^{r-1}=c^{r} c^{(b, r-1)}\left(c^{(b)}\right)^{r-1}>0 .
\end{aligned}
$$

Эта формула дает приведенные в утверждении теоремы 1 оценки для $\lambda$ и для вероятности совместности случайной системы уравнений.

Перейдем к оценке надежности алгоритма Al. При условии (2) почти все реализации случайного гиперграфа $M G(S)$ состоят из гипердеревьев, имеющих не более $r$ ребер, а вид минимальных несовместных подсистем, соответствующих гипердеревьям, приведен в утверждении п.(а) леммы 1. Из описания алгоритма Al следует, что он ищет именно такие несовместные подсистемы. Из предыдущего доказательства теоремы 1 следует, что сумма вероятностей реализаций случайной системы уравнений $S$, содержащих несовместные подсистемы, соответствующие гипердеревьям, приведенным в утверждении п. (а) леммы 1 , эквивалентна предельному значению вероятности $1-P_{c}(S)$ несовместности случайной системы уравнений $S$. Следовательно, предельная надежность алгоритма $\mathrm{Al}$ тоже эквивалентна этой величине. Теорема 1 доказана.

Доказательство теоремы 2. Пусть выполнены условия $R^{(0)} R^{(1)}>0$ и $c^{(0)}+c^{(1)}>0$. Почти все реализации случайного гиперграфа $M G(S)$ при условии $M=o(n)$ состоят из гипердеревьев. Из леммы 2 следует, что при условии $c^{(0)}+c^{(1)}=0$ подсистемы, соответствующие гипердеревьям, совместны. Поэтому при $M=o(n)$ и $c^{(0)}+c^{(1)}=0$ почти все реализации случайной системы уравнений $S$ совместны при всех значениях величины $R^{(0)} R^{(1)}$. В соответствии с леммой 3 при $M / n \rightarrow \infty, R^{(0)} R^{(1)}>0$ справедлива оценка $\mathbf{E} \zeta_{n}=o(1)$, из которой с учетом неравенства $\mathbf{P}\left(\zeta_{n} \geqslant 1\right) \leqslant \mathbf{E} \zeta_{n}$ следует, что почти все 
реализации случайной системы уравнений $S$ несовместны независимо от значения величины $c^{(0)}+c^{(1)}$. Таким образом, выполнения условия $\left\{R^{(0)} R^{(1)}>0\right\} \cap\left\{c^{(0)}+c^{(1)}=0\right\}$ достаточно для наличия у случайной системы уравнений $S$ пороговой функции совместности $n$.

Необходимость этого условия следует из того, что при его отрицании, имеющем вид $\left\{R^{(0)} R^{(1)}=0\right\} \cup\left\{\left\{R^{(0)} R^{(1)}>0\right\} \cap\left\{c^{(0)}+c^{(1)}>0\right\}\right\}$, случайная система уравнений $S$ не имеет пороговой функции совместности, равной $n$. В первом случае $R^{(0)} R^{(1)}=0$ для некоторого $b \in B$ справедливо равенство $R^{(b)}=0$, откуда, в соответствии с п. (б) леммы 1 , все реализации случайной системы уравнений $S$ имеют решение $\mathbf{b}$, и случайная система уравнений $S$ не имеет никакой пороговой функции совместности. Если же $R^{(0)} R^{(1)}>0$, $c^{(0)}+c^{(1)}>0$, то в соответствии с теоремой 1 случайная система уравнений $S$ имеет пороговую функцию совместности, которая не равна $n$. Теорема 2 доказана.

Обозначим через $\zeta_{n}$ число решений случайной системы уравнений $S$.

Лемма 3. При условии $R^{(0)} R^{(1)}>0$ существвует константа $\beta>0$ такая, что при $M-n \beta \rightarrow \infty$ справедлива оценка $\mathbf{E} \zeta_{n}=o(1)$.

Доказательство. Случайную систему уравнений $S$ можно рассматривать как вектор из $M$ случайных уравнений, имеющих одинаковое распределение. Поэтому вероятность того, что вектор $\left(b_{1}, \ldots, b_{n}\right)$ с компонентами из $B$ является решением для одного такого случайного уравнения, одинакова для каждого из этих $M$ уравнений. Эта вероятность одинакова для всех векторов с одинаковым числом $n_{1}$ равных 1 компонент, обозначим ее через $P\left(n_{1}\right)$. Число таких векторов равно $\left(\begin{array}{c}n \\ n_{1}\end{array}\right)$, поэтому

$$
\mathbf{E} \zeta_{n}=\sum_{n_{1} \leqslant n / 2}\left(\begin{array}{c}
n \\
n_{1}
\end{array}\right)\left(P\left(n_{1}\right)\right)^{M}+\sum_{n_{1}>n / 2}\left(\begin{array}{c}
n \\
n_{1}
\end{array}\right)\left(P\left(n_{1}\right)\right)^{M}
$$

Из условия $R^{(0)} R^{(1)}>0$ следует существование наборов $\left(i_{0}, j_{0}, a_{0}\right),\left(i_{1}, j_{1}, a_{1}\right)$, удовлетворяющих условию (3). Тогда при $n_{1} \leqslant n / 2$

$$
P\left(n_{1}\right) \leqslant 1-c_{i_{0}, j_{0}, a_{0}}\left(n-n_{1}\right)_{i_{0}} /(n)_{i_{0}} \leqslant 1-c_{i_{0}, j_{0}, a_{0}} / 2^{i_{0}}=\delta_{0}<1,
$$

где $c_{i_{0}}, j_{0}, a_{0}-$ вероятность появления в реализациях случайной системы $S$ уравнения вида (1) при $(i, j, a)=\left(i_{0}, j_{0}, a_{0}\right)$, а $\left(n-n_{1}\right)_{i_{0}} /(n)_{i_{0}}-$ нижняя оценка вероятности несовместности такого уравнения при $n_{1} \leqslant n / 2$. Аналогично при $n_{1}>n / 2$ получаем оценку

$$
P\left(n_{1}\right) \leqslant 1-c_{i_{1}, j_{1}, a_{1}}\left(n_{1}\right)_{i_{1}} /(n)_{i_{1}} \leqslant 1-c_{i_{1}, j_{1}, a_{1}} / 2^{i_{1}}=\delta_{1}<1,
$$

из которой следует, что

$$
P\left(n_{1}\right) \leqslant \delta<1, \quad \delta=\max \left\{\delta_{0}, \delta_{1}\right\},
$$

и

$$
\mathbf{E} \zeta_{n} \leqslant \sum_{0 \leqslant n_{1} \leqslant n}\left(\begin{array}{c}
n \\
n_{1}
\end{array}\right) \delta^{M} \leqslant 2^{n} \delta^{M}
$$

Поэтому $\mathbf{E} \zeta_{n}=o(1)$ при условии $M-n \beta \rightarrow \infty, \beta=\log _{1 / \delta} 2$. Отсюда следует утверждение леммы 3 . 
Более точные оценки $\beta$ при более строгих условиях на вектор вероятностей $\bar{c}$ получены в работах Г. В. Балакина, В. Ф. Колчина, В. А. Копытцева, В. И. Хохлова, исследовавших пороговый эффект для среднего числа решений случайных систем уравнений с независимыми левыми и правыми частями.

Доказательство теоремы 3. Каждая из величин $R^{(0)} R^{(1)}$ и $c^{(0)}+c^{(1)}$ может быть либо положительна, либо равна нулю. При $R^{(0)} R^{(1)}=0$ случайная система уравнений $S$ имеет решение $\mathbf{0}$ и/или 1 и, следовательно, не имеет пороговой функции совместности. Рассмотрим случай $R^{(0)} R^{(1)}>0$. Если $c^{(0)}+c^{(1)}=0$, то в соответствии с теоремой 2 случайная система уравнений $S$ имеет пороговую функцию совместности $n$. Если же $c^{(0)}+c^{(1)}>0$, то найдется $b \in B$ такое, что выполняются соотношения $c^{(b)}>0$, $1 \leqslant R^{(b)} \leqslant m$, и в соответствии с теоремой 1 случайная система уравнений $S$ имеет пороговую функцию совместности $n^{1-1 / r}, 2 \leqslant r=R^{(b)}+1 \leqslant m+1$.

Рассмотрены все варианты значений величин $R^{(0)} R^{(1)}$ и $c^{(0)}+c^{(1)}$ : при $R^{(0)} R^{(1)}=0$ случайная система уравнений $S$ не имеет пороговую функцию совместности, а при $R^{(0)} R^{(1)}>0$ она имеет пороговую функцию совместности указанного в утверждении теоремы 3 вида. Теорема 3 доказана.

\section{4. Примеры}

Далее под значениями 0 и 1 неизвестных систем уравнений понимаются элементы конечного поля из двух элементов, $\oplus-$ операция сложения в этом поле. В первых пяти примерах уравнения (1) случайной системы $S$ имеют вид

$$
f_{t d, j}\left(x_{s_{1}}, x_{s_{2}}, \ldots, x_{s_{d k}}\right)=\sum_{k=1}^{t} f_{d, j}\left(x_{s_{d(k-1)+1}}, x_{s_{d(k-1)+2}}, \ldots, x_{s_{d(k-1)+d}}\right)=a,
$$

$t=1, \ldots, h$, где $h=\lfloor m / d\rfloor, f_{t d, j}-$ функция, равная сумме в поле действительных чисел $t$ булевых функций $f_{d, j}$ из $\Phi_{d}, d \geqslant 1$, правая часть $а$ уравнения вида (4) принимает целые значения $0,1, \ldots, t$. Уравнение (4) при $1 \leqslant a \leqslant t-1$ не имеет однозначно определяющихся переменных, а при $a=0$ и $a=t$ равносильно системе из $t$ уравнений

$$
f_{d, j}\left(x_{s_{d(k-1)+1}}, x_{s_{d(k-1)+2}}, \ldots, x_{s_{d(k-1)+d}}\right)=b, \quad k=1,2, \ldots, t,
$$

где $b=0$ и $b=1$ соответственно.

Пример 1. Функции в (4) имеют вид $f_{1,1}\left(y_{1}\right)=y_{1}, d=1$. В этом случае $c^{(0)}=\sum_{k=1}^{m}\left(k c_{k, 1,0}\right), c^{(1)}=\sum_{k=1}^{m}\left(k c_{k, 1, k}\right) ; R^{(0)}=0$ при $\sum_{k=1}^{m} c_{k, 1,0}=1, R^{(1)}=0$ при $\sum_{k=1}^{m} c_{k, 1, k}=1$. Пороговая функция совместности равна $\sqrt{n}$ при $c^{(0)} c^{(1)}>0$; равна $n^{1-1 / r}, r=3, \ldots, m+1$ при $c^{(0)}>0, \sum_{k=1}^{m}\left(c_{k, 1, k-r+3}+\ldots+c_{k, 1, k}\right)=0$, $\sum_{k=1}^{m} c_{k, 1, k-r+2}>0$ или при $c^{(1)}>0, \sum_{k=1}^{m}\left(c_{k, 1,0}+c_{k, 1,1}+\ldots+c_{k, 1, r-3}\right)=0$, $\sum_{k=1}^{m} c_{k, 1, r-2}>0$; равна $n$ при $c^{(0)}+c^{(1)}=0$. Совместность близкой случайной системы уравнений с пороговой функцией $\sqrt{n}$ оценивалась В. А. Копытцевым, случай $|B| \geqslant 2$ рассмотрен в примере 4.1 статьи [13], при $c_{m}=1, m=o(\sqrt{n})$ в [2].

Пример 2. Функции в (4) имеют вид $f_{d, 2}\left(y_{1}, \ldots, y_{d}\right)=y_{1}\left(y_{2} \oplus \ldots \oplus y_{d}\right), d \geqslant 3$. В этом случае $c^{(0)}=0, c^{(1)}=\sum_{k=1}^{h}\left(k c_{k d, 2, k}\right) ; R^{(0)}=0$ при четных $d$ и $\sum_{k=1}^{h} c_{k d, 2,0}=1$; $R^{(0)}=R^{(1)}=0$ при нечетных $d$ и $\sum_{k=1}^{h} c_{k d, 2, k}=1$ или $\sum_{k=1}^{h} c_{k d, 2,0}=1$. Если $d$ четно и $c^{(1)}>0, c^{(1, d)}=\sum_{k=1}^{h}\left(k c_{k d, 2,0}\right)>0$, то $R^{(1)}=d$ и пороговая функция 
совместности равна $n^{1-1 /(d+1)}$. Если $d$ нечетно и $c^{(1)}>0, c^{(1, d-1)}=\sum_{k=1}^{h}\left(k c_{k d, 2,0}\right)>0$, то $R^{(1)}=d-1$ и пороговая функция совместности равна $n^{1-1 / d}$. Пороговая функция совместности также может иметь вид $n^{1-1 /(1+k d)}$ и $n^{1-1 /(1+k(d-1))}, k=2, \ldots, h$.

Пример 3. Функции в (4) имеют вид $f_{d, 3}\left(y_{1}, \ldots, y_{d}\right)=\bar{y}_{1}\left(y_{2} \oplus \ldots \oplus y_{d}\right), d \geqslant 3$. Здесь $c^{(1)}=0, c^{(0)}=\sum_{k=1}^{h}\left(k c_{k d, 3, k}\right) ; R^{(0)}=R^{(1)}=0$ при $\sum_{k=1}^{h} c_{k d, 3,0}=1$. Пороговая функция совместности равна $n^{1-1 / d}$ тогда и только тогда, когда $c^{(0)}>0$, при этом $R^{(0)}=d-1, r=d, c^{(0, d-1)}=c^{(0)}$.

Пример 4. Функции в (4) имеют вид $f_{d, 4}\left(y_{1}, \ldots, y_{d}\right)=y_{1} \vee \ldots \vee y_{d}, d \geqslant 2$. Здесь $c^{(1)}=0, c^{(0)}=d \sum_{k=1}^{h}\left(k c_{k d, 4,0}\right) ; R^{(0)}=0$ при $\sum_{k=1}^{h} c_{k d, 4,0}=1 ; R^{(1)}=0$ при $\sum_{k=1}^{h} c_{k d, 4, k}=1$. Если $c^{(0, d)}=\sum_{k=1}^{h}\left(k c_{k d, 4, k}\right)>0, c^{(0)}>0$, то пороговая функция совместности равна $n^{1-1 /(d+1)}$. Вероятность совместности такой случайной системы уравнений с пороговой функцией $n^{2 / 3}$ при $c_{2}=1, m=d=2$ оценивалась Г. В. Балакиным в [1], при $d=2, m=2 h, c_{m}=1-$ В. А. Копытцевым.

Примеры 2-4 рассмотрены в [12] при $m=d, c_{m}=1$.

Пример 5. Функция $f_{d, j}$ в (4) не имеет однозначно определяющихся переменных. При $R^{(0)} R^{(1)}>0$ пороговая функция совместности равна $n$.

Пример 6. В случайной системе булевых уравнений $S$ вероятность появления линейного уравнения с функцией $y_{1} \oplus y_{2} \oplus \ldots \oplus y_{i}$ равна $c_{i, 5}, i=2, \ldots, m$, причем $c_{2,5}+\ldots+c_{m, 5}=1$. Здесь $c^{(0)}=c^{(1)}=0 ; R^{(1)}=0$ при $\sum_{k=1}^{(m-1) / 2} c_{2 k+1,5,1}=1$ или $\sum_{k=1}^{m / 2} c_{2 k, 5,0}=1 ; R^{(0)}=0$ при $\sum_{i=2}^{m} c_{i, 5,0}=1$. В остальных случаях пороговая функция совместности равна $n$.

Пример 7. Функции в уравнениях случайной системы $S$ булевы и имеют вид элементарных симметрических многочленов $\sum_{1 \leqslant i_{1}<i_{2}<\ldots<i_{k} \leqslant m} y_{i_{1}} y_{i_{2}} \ldots y_{i_{k}}$, где $2 \leqslant k \leqslant m-1$, $m \geqslant 3$. Если правые части всех уравнений равны 0 , то $R^{(0)}=0$. Пороговая функция совместности равна $n$ тогда и только тогда, когда $R^{(0)} R^{(1)}>0$.

\section{Список литературы}

1. Балакин Г. В., Графы систем двучленных уравнений с булевыми неизвестными. Теория вероятностей и ее применения (1995) 40, №2, 241-259.

2. Балакин Г. В., Системы случайных уравнений над конечным полем. Tруды по дискретной математике (1998) 2, 21-37.

3. Балакин Г. В., Колчин В. Ф., Хохлов В. И., Гиперциклы в случайном гиперграфе. Дискретная математика (1991) 3, №3, 102-108.

4. Зыков А. А., Гиперграфы. Успехи матем. наук (1974) 29, №6, 89-154.

5. Колчин В. Ф., Системы случайных уравнений. МИЭМ, Москва, 1988.

6. Колчин В. Ф., Вероятность совместности одной системы случайных уравнений специального вида. Труды по дискретной математике (2000) 3, 139-146.

7. Колчин В. Ф., Случайные графы. Физматлит, Москва, 2004.

8. Колчин В. Ф., Хохлов В. И., О числе циклов в случайном неравновероятном графе. Дискретная математика (1990) 2, №3, 137-145.

9. Колчин В. Ф., Хохлов В. И., Пороговый эффект для систем случайных уравнений специального вида. Дискретная математика (1995) 7, №4, 29-39. 
10. Копытцев В. А., О некоторых результатах, связанных с анализом систем случайных уравнений. Вестник ИКСИ, Сер. К, Спеи. вып. (2003) 71-75.

11. Сачков В. Н., Случайные неравновероятные покрытия и функциональные уравнения. Труды по дискретной математике (2002) 5, 205-218.

12. Шаповалов А. В., Вероятность совместности случайных систем булевых уравнений. Дискретная математика (1995) 7, №3, 146-159.

13. Шаповалов А. В., Пороговые функции совместности случайных систем уравнений. Труды по дискретной математике (2006) 9, 377-400.

14. Bollobás B., Random graphs. Academic Press, London, 1985.

15. Erdős P., Rényi A., On the evolution of random graphs.. Publ. Math. Inst. Hung. Acad. Sci., Ser. A (1960) 5, 17-61.

Статья поступила 10.07.2008. 